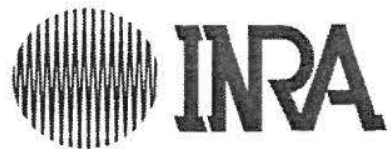

STATION D'ECONOMIE ET SOCIOLOGIE RURALES DE RENNES

UNITE POLITIQUE AGRICOLE ET MODELISATION 65 , rue de St-Brieuc - 35042 Rennes cedex - France tél. (33) 99.28.53.82 - fax. (33) 99.28.53.80

12èmes journées de micro-économie appliquée

1-2 Juin 1995

Cerdi, Clermont-Ferrand
NSIIIUT NATIONAL DE LA RECHERCHE AGHUNGUmuUE Station d'Economie en Sociologie Purales DOCUMENTATION

65. Rue de St Brieuc 35042 RENNES CEDEX Tél. : 99.28 .54 .08 et 09

\title{
Le problème de la qualité du café indonésien : une interprétation micro-économique
}

\author{
Catherine Benjamin, Chantal Le Mouël
}

I..N.R.A.-E.S.R. - Institut National de la Recherche Agronomique Station d'Economie et Sociologie Rurales de Rennes - Unité PAM 
L'objectif de ce papier est de comprendre pourquoi la valorisation de la qualité du café (par le biais d'un système de prix différencié) ne conduit pas systématiquement les producteurs de café à effectuer une cueillette sélective. Il s'agit, donc, dans un premier temps, de mettre en évidence les facteurs qui conditionnent la décision des producteurs quant au choix de leur mode de cueillette des cerises de café. La seconde étape consiste à tenter d'expliquer le comportement dichotomique des récolteurs par rapport à la technique de cueillette utilisée. De façon plus précise, on observe sur l'échantillon de producteurs indonésiens dont nous disposons que les deux modes de cueillette ne sont jamais utilisés simultanément. Il s'agit donc de s'interroger sur ce type de comportement des producteurs qui consiste, une fois le mode de cueillette choisie (sélective ou non sélective) à l'appliquer à toute leur surface caféière cultivée.

Le modèle d'analyse proposé repose sur la théorie micro-économique du ménage agricole. Ainsi, le cadre théorique utilisé prend en compte dans un cadre simultané les décisions de consommation et de production du ménage (Nakajima, 1966). De plus, le programme de comportement intègre de manière explicite le rôle de la contrainte de crédit. Enfin, le modèle est développé dans un cadre intertemporel.

L'interprétation du comportement du ménage par rapport au choix de la méthode de cueillette repose alors sur le constat de l'existence d'imperfections sur le marché indonésien du capital. Une conséquence du dysfonctionnement de ce marché est la possible apparition d'un rationnement sur le montant du crédit disponible pour le ménage.

Une application économétrique est, ensuite, réalisée à partir d'une enquête réalisée en 1992 auprès de 115 producteurs d'une des principales régions productrices de café en Indonésie. Dans l'échantillon, $60 \%$ des producteurs réalisent une cueillette sélective. Un modèle expliquant la pratique ou non d'une cueillette sélective est estimé à partir des informations sur les caractéristiques du ménage, de l'exploitation, sur le prix reçu par le producteur et sur l'existence de crédit. Les résultats économétriques permettent de quantifier les effets des différents facteurs et en particulier ceux relevant de la contrainte de crédit sur la probabilité du choix de la méthode de cueillette.

Le plan du papier est le suivant. La deuxième section précise les sources de l'existence d'imperfections sur le marché du capital, puis étudie leur rôle sur le comportement du ménage. L'application économétrique et l'analyse de ses résultats font l'objet de la troisième section. Enfin, la quatrième section présente quelques éléments de conclusion. 


\section{Conséquences des imperfections sur le marché du capital}

L'objet de cette section est d'analyser de manière théorique l'impact du crédit sur les activités de production du ménage.

Tout d'abord, il s'agit de préciser comment les contraintes de crédit découlent d'imperfections existant sur le marché du capital. Le premier paragraphe rappelle les deux principales sources de ces imperfections. Le second paragraphe est consacré à la présentation de la démarche analytique et du modèle de comportement retenu. Enfin, le troisième paragraphe analyse l'impact de la contrainte de crédit sur les décisions du ménage.

\subsection{Imperfections sur le marché du capital et contraintes de crédit}

Le crédit est un élément important dans le système de production agricole. Il permet aux producteurs de couvrir les besoins en liquidité créés par les cycles de production agricole qui caractérisent l'agriculture. En effet, ces différentes phases correspondant notamment à la préparation des surfaces, à la plantation, aux cultures et aux récoltes de produits sont réalisées sur une période de plusieurs mois durant laquelle peu d'argent liquide est gagné (Feder et al., 1990). Les liquidités sont reçues après récolte. Dans le cas d'absence d'un marché du crédit ou d'imperfections sur ce marché, les exploitants agricoles doivent maintenir une réserve financière pour assurer la consommation et les dépenses de production des périodes précédant la récolte.

Ces imperfections peuvent alors provoquer un rationnement sur le montant de la dette que le ménage souhaite contracter. Le dysfonctionnement de ce marché peut apparaître sous différentes formes. Tout d'abord, il peut exister des coûts de transaction liés à la réalisation d'emprunt. Ces coûts de transaction se traduisent, alors, par un écart entre le taux d'intérêt rémunérant l'épargne du ménage et le taux d'intérêt correspondant au coût de l'emprunt (Phimister, 1993). Un deuxième type d'imperfections du marché du capital peut apparaître en raison de l'information imparfaite détenue par les opérateurs participant au marché. Ainsi, l'intermédiaire financier qui prête au ménage agricole peut être incertain des capacités de remboursement de ce dernier. L'asymétrie d'information apparaissant dans le contrat établi entre le producteur et l'intermédiaire empêche le bon fonctionnement du marché (Cosci, 1993). En effet, cette incertitude sur la qualité de l'emprunteur provoque l'exigence de garanties contractuelles. Du fait de l'existence d'une asymétrie d'information, le taux d'intérêt n'est pas suffisant pour garantir la seule sélection des emprunteurs possédant des capacités réelles de remboursement (Stigliss et Weiss, 1983). Il peut, alors, devenir optimal de rationner le crédit. En particulier, les ménages agricoles qui empruntent font face vraisemblablement à des restrictions du montant de leur endettement basées sur leurs caractéristiques financières et leurs avoirs. Dans ce papier, nous supposons l'existence d'une imperfection du marché du capital exprimée par l'existence d'une contrainte du niveau d'endettement. 


\subsection{Le modèle de comportement}

L'introduction d'une imperfection sur le marché du capital conditionne le cadre analytique utilisé. Par définition, les décisions de production du ménage agricole sont identiques aux solutions déterminées dans le programme de maximisation du profit quand il existe des marchés parfaits pour tous les biens (Singh et al., 1986). La défaillance d'un marché est une source de destruction de la séparabilité entre décisions de consommation et décisions de production. Pour capter cette éventuelle interdépendance, il est nécessaire de modéliser dans un cadre simultané les décisions de consommation et de production du ménage agricole (Singh et al., 1986). Aussi, le cadre théorique retenu dans ce papier est le modèle du ménage producteur et consommateur.

Pour décrire le comportement des producteurs de café, le raisonnement s'effectue dans le cadre d'un modèle intertemporel comprenant deux périodes : le présent représenté par la période 1 et le futur décrit par la période 2 . La période 2 caractérise la date où les grains de café sont mûrs. Les producteurs récoltant lors de cette période font donc une cueillette sélective. On suppose que l'exercice d'une cueillette sélective est valorisé, c'est-à-dire que le prix unitaire perçu par le producteur à la deuxième période est supérieur au prix unitaire reçu par le producteur quand il vend sa production à la première période. Dans ce modèle simplifié on admettra de plus qu'il n'y a pas d'inflation.

Le ménage est supposé maximiser son utilité intertemporelle sur deux périodes. Cette dernière est une fonction définie à partir de la consommation de biens et de services sur les deux périodes (notées respectivement $C_{1}$ et $C_{2}$ ) et des niveaux de travail familial sur l'exploitation correspondants (notés $L F_{1}$ et $L F_{2}$ ). La fonction d'utilité du ménage est supposée croissante strictement quasi-concave par rapport aux biens consommés, décroissante par rapport aux niveaux de travail et séparable de façon additive sur les deux périodes.

Sous ces hypothèses le programme de comportement du ménage s'écrit

$$
\begin{aligned}
& \max _{d_{l}, C_{i}, Y_{i}, L F_{i}, L H_{i}, t e r_{i}} U\left(L F_{1}, C_{1}\right)+\left(\frac{1}{1+\rho}\right) U\left(L F_{2}, C_{2}\right) \\
& i=1.2 \\
& L E I_{i}=T-L F_{i} \\
& p_{C_{l}} C_{l}=p_{I} Y_{l}-w L H_{l}-c_{\text {cter }}-d_{l}+d_{0}(I+r) \\
& p_{C_{2}} C_{2}=p_{2} Y_{2}-w L H_{2}-\text { cter }_{2}+d_{1}(1+r) \\
& Y_{i}=F\left(L_{i}, \text { ter }_{i}\right) \\
& (i=1,2) \\
& L_{i}=L F_{i}+L H_{i} \\
& (i=1,2) \\
& \text { Ter }=\text { ter }_{1}+\text { ter }_{2} \\
& d_{1} \geq-\bar{B} \\
& C_{i} \geq 0
\end{aligned}
$$




$$
\begin{aligned}
& L H_{i} \geq 0 \\
& L F_{i} \geq 0 \\
& \operatorname{ter}_{i} \geq 0 \\
& T, c, d_{o}, \text { Ter, } \bar{B} \text { fixés. }
\end{aligned}
$$

où $U$ définit la fonction d'utilité du ménage, $\rho$ est le taux d'escompte psychologique, $C_{i}(i=1,2)$ la consommation en biens et services dans la période $\mathrm{i}, p_{C_{t}}, p_{C_{2}}$ leurs prix respectifs et $L E I_{i}(i=1,2)$ le niveau de loisir de la période i. $T$ représente le temps total disponible du ménage.

Le montant de la dette ou d'épargne du ménage de la période 1 est noté $d_{1}$. Si la variable $d_{l}$ est négative, alors le ménage réalise un emprunt dans la période 1 au taux d'intérêt $r$, le remboursement intervenant dans la période 2. A l'inverse, si la variable $d_{l}$ est positive alors le ménage épargne dans la période 1 , les intérêts étant perçus dans la période 2 . La variable fixée $d_{0}$ définit le montant initial de l'endettement du ménage. On considère donc que cette variable est négative ou nulle. Son niveau peut correspondre aux dépenses effectuées lors de la période précédente pour la préparation et l'entretien des caféiers. De plus, dans un souci de simplification, on suppose qu'à la fin de la période étudiée, le ménage n'a pas de dette ni d'épargne.

Le paramètre $c$ désigne les coûts fixes et exogènes apparaissant quand il y a une récolte (correspondant principalement aux coûts de transports pour se rendre aux parcelles cultivées ${ }^{1}$ ). Ter, supposée fixée définit le niveau de surface qui a été cultivée en café et qu'il faudra récolter. Le niveau d'output produit à la période i est noté $Y_{i}, p_{i}$ le prix associé, la fonction $\mathrm{F}$ décrit la technologie de production qui dépend du niveau de travail employé sur l'exploitation $L_{i}(i=1,2)$ et de la surface récoltée $t e r_{i}$. La demande de travail de l'exploitation est couverte par du travail salarié $L H_{i}$ w étant le prix associé, et du travail familial $L F_{i}$.

La première contrainte (a) définit pour le ménage la contrainte de temps. Le temps disponible est réparti entre un travail sur l'exploitation et le loisir.

Les contraintes (b) et (c) sont les contraintes budgétaires associées respectivement à la période 1 et à la période 2. Pour chaque période, le revenu du ménage est la somme de la valeur de la production, diminuée du total des salaires versés au personnel salarié sur l'exploitation et des coûts fixes et exogènes. A ce revenu issu des activités de production est ajouté (respectivement enlevé) dans la période 1, le niveau de dette (respectivement d'épargne) réalisé au cours de cette période, le dernier terme négatif correspond au montant du remboursement du crédit de la période précédente. Dans la période 2, le ménage rembourse le (respectivement dispose du) montant de dette (respectivement d'épargne) réalisé dans la période 1 .

\footnotetext{
${ }^{1}$ Les producteurs indonésiens cultivent généralement plusieurs parcelles caféières dispersées et situées pour la plupart à plus de $0,5 \mathrm{~km}$ du domicile de l'exploitant (De Graaf, 1986 ; Budisentoso, 1990).
} 
Les contraintes (di) caractérisent la relation technique de production. La fonction de production est supposée deux fois différentiable et strictement quasi-concave. Le facteur travail est supposé être un facteur de production essentiel. De plus, la productivité marginale de la terre est supposée constante. Les deux facteurs de production sont supposés complémentaires.

Les contraintes (ei) définissent la demande de travail de l'exploitation comme une fonction additive du niveau de travail familial et du niveau de travail salarié. Cette écriture sous entend une substitution et une équivalence parfaite entre ces deux types de travail.

L'égalité (f) indique que le producteur a cultivé un montant de terre fixe. A chaque période, il choisit le niveau de superficie à récolter.

La contrainte (g) illustre l'imperfection du marché du capital. Au taux d'intérêt d'équilibre, le ménage agricole ne peut pas obtenir tout le crédit souhaité. Le ménage est rationné pour son niveau de dette ${ }^{2}$. On suppose que l'offre de crédit notée $\bar{B}$ qui définit le montant maximal que le ménage peut emprunter, dépend des terres possédées par le ménage (Feder, 1985).

Les autres contraintes sont les contraintes de positivité associées aux variables endogènes.

\subsection{L'impact du crédit sur le comportement de production du ménage}

Afin de simplifier l'analyse, on suppose qu'à l'optimum les quantités de biens et de services sont des solutions intérieures. Les prix des vecteurs consommations sont de plus pris comme numéraires. De plus, on note $M_{i}$ le revenu du ménage de la période i.

Les conditions du premier ordre s'écrivent alors

$$
\begin{aligned}
& -\left(\partial U / \partial L F_{1}\right) /\left(\partial U / \partial M_{1}\right)-p_{1}\left(\partial F / \partial L_{1}\right)-\mu_{1} /\left(\partial U / \partial M_{1}\right)=0 \\
& -(1 / 1+\rho)\left(\partial U / \partial L F_{2}\right) /\left(\partial U / \partial M_{2}\right)-\left(p_{2} / 1+\rho\right)\left(\partial F / \partial L_{2}\right)-\mu_{2} /\left(\partial U / \partial M_{2}\right)=0 \\
& p_{1}\left(\partial F / \partial L_{1}\right)-w+\varepsilon_{1} /\left(\partial U / \partial M_{1}\right)=0 \\
& \left(p_{2} / 1+\rho\right)\left(\partial F / \partial L_{2}\right)-(w / 1+\rho)+\varepsilon_{2} /\left(\partial U / \partial M_{2}\right)=0 \\
& -\left(\partial U / \partial M_{1}\right)+(1+r / 1+\rho)\left(\partial U / \partial M_{2}\right)+\lambda_{1}=0 \\
& p_{1}\left(\partial F / \partial t e r_{1}\right)-c+\varsigma_{1} /\left(\partial U / \partial M_{1}\right)=0 \\
& \left(p_{2} / 1+\rho\right)\left(\partial F / \partial t e r_{2}\right)-(c / 1+\rho)+\varsigma_{2} /\left(\partial U / \partial M_{2}\right)=0
\end{aligned}
$$

\footnotetext{
2 Dans le contexte indonésien, deux raisons justifient ce rationnement: i) le problème d'information sur la qualité de l'emprunteur, et ii) le fait que certains intermédiaires financiers ne sont que des agents de commercialisation localisés dans des petits villages et disposent de peu de fonds propres.
} 
où $\mu_{i}$ (respectivement $\varepsilon_{i}$ ) est le multiplicateur de Lagrange associé à la contrainte de positivité sur les niveaux de travail familial (respectivement travail salarié) employés sur l'exploitation pendant la période $\mathrm{i}, \lambda_{1}$ le multiplicateur associé à la contrainte limitant le crédit associé au ménage dans la période $1, \varsigma_{i}$ est le multiplicateur associé à la contrainte de positivité de la terre pour la période $\mathrm{i}$.

Les équations (2) et (3) montrent qu'à l'optimum le travail familial sur l'exploitation est positif si le taux marginal de substitution du revenu au travail familial est égal à la productivité marginale en valeur de ce travail.

Les équations (4) et (5) caractérisent les situations d'utilisation ou non d'emploi de travail salarié sur l'exploitation selon que la productivité marginale de ce facteur, en valeur, est égale ou supérieure à son coût.

L'équation (6) montre que quand le ménage agricole est contraint au niveau de son crédit l'utilité marginale du revenu de la première période est inférieure à l'utilité marginale du revenu de la seconde période.

Les équations (7) et (8) montrent que quand il y a récolte la productivité marginale de la terre, en valeur, est égale aux coûts fixes associés à cette récolte.

Différents régimes de travail peuvent être définis suivant la nullité et positivité des multiplicateurs $\mu_{i}$ et $\varepsilon_{i}$. Chacun de ces régimes se dédouble suivant la saturation ou non de la contrainte de crédit. Certains de ces régimes se révèlent être des solutions impossibles. Les conditions du premier ordre et la non-négativité des multiplicateurs entraînent les propositions suivantes.

Proposition 1. Pour certaines valeurs du montant initial de dette, le ménage ne peut pas effectuer une cueillette sélective.

On étudie le cas où le ménage effectue une cueillette sélective, c'est à dire récolte uniquement en seconde période. Ce cas correspond au régime où les multiplicateurs $\mu_{l}$ et $\varepsilon_{l}$ sont positifs tandis que les multiplicateur $\mu_{2}$ et $\varepsilon_{2}$ sont nuls. Dans ce cas, la demande de travail en première période est nulle $\left(L_{l}=0\right)$ et est positive en seconde période $\left(L_{2}>0\right)$. Les conditions du premier ordre s'écrivent $p_{1} \frac{\partial F}{\partial L H_{1}}<w$ et $p_{2} \frac{\partial F}{\partial L H_{2}}=w$. Les contraintes budgétaires deviennent alors

$$
\begin{aligned}
& p_{C_{1}} C_{1}=-d_{1}+d_{o}(1+r) \\
& p_{C 2} C_{2}=d_{1}(1+r)+p_{2} F\left(L_{2}\right)-w L H_{2}-c_{t e r}
\end{aligned}
$$

La condition pour que la valeur de la consommation soit positive dans la première période est $d_{0} \geq \frac{-\bar{B}}{1+r}$. 
On déduit de cette inégalité l'information suivante : si le ménage a emprunté dans la période précédente un montant compris entre $\frac{\bar{B}}{1+r}$ et $\bar{B}$ (en valeur absolue) alors l'offre de travail ne peut pas être nulle dans la première période. Le ménage doit récolter lors de la première période pour pouvoir couvrir ses dépenses.

Cette première proposition montre donc clairement que la contrainte de crédit peut contrecarrer l'effet attendu d'une politique de prix différencié. En effet, on voit ici que cette contrainte peut amener les producteurs à effectuer une cueillette non sélective (i.e., récolte au cours de la période 1) même si la qualité du café est valorisée par le biais d'un système de prix différencié (i.e., $p_{2}>p_{1}$ ).

Proposition 2. Si l'exploitation emploie du travail salarié, cette utilisation n'aura lieu qu'en deuxième période.

Le régime de travail caractérisant l'emploi de travail salarié sur l'exploitation pour les deux périodes apparaît quand les multiplicateurs $\varepsilon_{1}$ et $\varepsilon_{2}$ sont nuls. Dans ce cas, les équations (3) et (4) s'écrivent

$$
\begin{aligned}
& p_{l}\left(\partial F / \partial L_{1}\right)-w=0 \\
& \left(p_{2} / 1+\rho\right)\left(\partial F / \partial L_{2}\right)-(w / 1+\rho)=0
\end{aligned}
$$

Or on suppose que la qualité du café est valorisée c'est à dire que $p_{2}>p_{1}$. Les deux équations de ce système sont donc incompatibles. Le régime de travail où il y a du travail salarié sur l'exploitation uniquement en période 1 apparaît quand le multiplicateur $\varepsilon_{I}$ est nul, soit

$$
\begin{aligned}
& p_{1}\left(\partial F / \partial L_{1}\right)-w=0 \\
& \left(p_{2} / 1+\rho\right)\left(\partial F / \partial L_{2}\right)<(w / 1+\rho)
\end{aligned}
$$

Ce cas est également impossible pour la même raison que précédemment.

Cette seconde proposition montre que l'utilisation du travail salarié liée à la comparaison de la productivité marginale en valeur au coût du travail salarié n'est effectuée que s'il y a cueillette sélective. Autrement dit, si les ménages sont contraints sur le crédit ils n'emploient pas de travail salarié (cette proposition est vraie quand la qualité du café est valorisée par le biais d'un système de prix différencié). 
Proposition 3. Quand l'exploitation n'emploie pas de travail salarié la demande de travail de l'exploitation ne peut pas être positive sur les deux périodes.

Le régime de travail où la demande de travail de l'exploitation est positive sur les deux périodes correspond au cas où $L F_{1}$ et $L F_{2}$ sont non nuls. On récolte sur les deux périodes. Dans ce cas, les équations (7) et (8) s'écrivent

$$
\begin{aligned}
& p_{l}\left(\partial F / \partial t e r_{1}\right)-c=0 \\
& \left(p_{2} / 1+\rho\right)\left(\partial F / \partial t e r_{2}\right)-(c / 1+\rho)=0
\end{aligned}
$$

or on suppose que la qualité du café est valorisée c'est à dire que $p_{2}>p_{1}$. Les deux équations de ce système sont donc incompatibles. Le niveau de terre cultivée ne peut donc pas être positif dans les deux périodes.

Cette proposition est directement liée à la question du comportement dichotomique des producteurs indonésiens évoqué lors de l'introduction. Elle signifie en effet qu'en l'absence de travail salarié, une fois le choix de cueillette effectué (sélective ou non) ellappliquée à toutes les parcelles cultivées (i.e., on ne peut pas observer l'emploi de travail familial simultanément sur les deux périodes).

Les propositions précédentes montrent clairement que le choix d'une cueillette sélective (i.e. la décision de récolter les cerises de café au cours de la période 2) dépend de deux facteurs essentiels : i) la valeur de la productivité marginale du travail au cours de la période 2 dépend et ii) le niveau de la contrainte de crédit à laquelle le ménage fait face. Ces deux facteurs permettent, par conséquent de définir un critère de cueillette sélective. L'ensemble des variables ayant une influence sur ces deux facteurs joue sur la probabilité qu'un ménage adopte la méthode de cueillette sélective. La mise en oeuvre d'un modèle liant la probabilité d'adoption de la méthode de cueillette sélective d'une part à diverses variables exogènes d'autre part fait l'objet de la section suivante. L'estimation d'un tel modèle permet alors de mesurer l'impact de chacune des variables exogènes et en particulier de celles ayant une influence sur la contrainte de crédit du ménage, sur la probabilité de choix d'une cueillette sélective. 


\section{Spécification économétrique et données}

La résolution du programme de comportement permet de définir les conditions d'exercice d'une cueillette sélective. Ces conditions sont présentées dans le premier paragraphe. Le second paragraphe est consacré à la description de l'échantillon de ménages indonésiens disponible et au choix des variables explicatives. Les résultats d'estimation sont analysés dans le troisième paragraphe. Enfin, le quatrième paragraphe propose une voie alternative pour une politique d'amélioration de la qualité du café en Indonésie.

\subsection{Critère d'exercice d'une cueillette sélective}

Le critère d'exercice d'une cueillette sélective est défini par les conditions du premier ordre du programme de maximisation de l'utilité intertemporelle du ménage exposé dans la section précédente. Ce critère s'écrit de la façon suivante:

$$
\begin{aligned}
& L_{2}>0 \text { et } L_{1}=0 \text { si } p_{2} \partial F / \partial L_{2} \geq w \text { et } d_{0}>-\bar{B} / 1+r \\
& L_{2}=0 \text { sinon }
\end{aligned}
$$

On suppose que la valeur de la productivité marginale du travail ${ }^{3}$ est définie de façon linéaire par rapport aux variables explicatives. Soit

$$
p_{2} \partial F / \partial L_{2}=X \beta_{h}+\varepsilon_{h}
$$

où $\mathrm{X}$ est le vecteur ligne des variables explicatives intervenant dans la fonction définissant la valeur de la productivité marginale du travail, $\beta_{h}$. est le vecteur des paramètres correspondants et $\varepsilon_{h}$ le terme d'erreur.

La variable $\bar{B}$ correspond aux garanties que le ménage agricole peut apporter à l'intermédiaire financier. Elle est définie comme une fonction positive des dotations en terre du ménage (Feder, 1985). Soit $\bar{B}=B(A), \quad \partial B / \partial A>0$.

La variable $d_{0}$ est le montant initial de dettes du ménage et est fonction du coût des intrants utilisés pour la préparation et l'entretien des caféiers et de la terre détenue par le ménage. On peut supposer que $d_{0}$ diminue avec le prix des inputs. En revanche, l'effet d'une augmentation de la surface détenue a un effet indéterminé sur le niveau de la dette. En effet, elle peut accroître l'épargne ou le niveau d'endettement.

Soit $\mathrm{D}$ une variable muette telle que $\mathrm{D}=1$ si le ménage réalise une cueillette sélective, 0 sinon. La règle de choix peut alors s'exprimer en fonction des variables explicatives, des paramètres à estimer et des termes d'erreur de la manière suivante suivante :

3 Dnas l'échantillon étudié, toutes les exploitations utilisent du travail salarié. 


$$
\begin{aligned}
\operatorname{Pr}\left(L_{2}>0\right) & =\operatorname{Pr}(D=1)=\operatorname{Pr}\left(X \beta_{h}+\varepsilon_{h} \geq w \text { et } d_{0}>-\bar{B} / 1+r\right) \\
& =\operatorname{Pr}\left(\varepsilon_{h}<X \beta_{h}-w \text { et } d_{0}>-\bar{B} / 1+r\right)
\end{aligned}
$$

La probabilité d'effectuer une cueillette sélective dépend donc de toutes les variables exogènes qui influencent la productivité marginale du travail et des variables exogènes qui définissent la contrainte de crédit. Les variables qui contribuent à accroître la productivité marginale du travail, toutes choses égales par ailleurs, augmentent la probabilité d'effectuer une cueillette sélective si le ménage n'est pas contraint. Ainsi, une augmentation de la superficie cultivée en café (la variable Ter) accroît la probabilité d'effectuer une cueillette sélective uniquement quand le ménage n'est pas contraint. En revanche, l'effet de l'augmentation de la surface totale possédée par la ménage a un impact indéterminé.

L'application économétrique que nous avons adoptée est une approche directe ${ }^{4}$ en estimant sous la forme d'un modèle probit dichotomique la probabilité d'effectuer une récolte sélective, les variables exogènes retenues dans la spécification sont précisées dans le prochain paragraphe.

\subsection{Choix des variables explicatives et spécification du modèle économétrique}

L'étude empirique est basée sur des données issues d'une enquête effectuée de juillet à octobre 1992 dans une province du sud de l'île de Sumatra. Cette région correspond à un des principaux lieux de production de café en Indonésie. De plus, la production caféière issue de cette région souffre, d'après les données du ministère du commerce indonésien, d'un important problème de qualité.

L'échantillon comprend 115 ménages sélectionnés de façon aléatoire, à partir de trois villages. Dans ce groupe, 70 producteurs de café (soit $60 \%$ des producteurs) réalisent une cueillette sélective. L'information disponible comprend des données liées aux activités de production (taille de l'exploitation, superficie allouée au café, nombre de parcelles cultivées, prix perçu par le producteur) aux caractéristiques du chef d'exploitation (âge, expérience, niveau d'éducation) et aux caractéristiques financières des ménages. Nous avons retenu dans la spécification finale les seules variables significatives. Une définition précise des différentes variables retenues dans l'estimation est présentée dans le tableau $1^{5}$.

\footnotetext{
4 Nous utilisons donc l'équation (17) seulement pour repérer les variables jouant sur les deux inégalités conditionnant le comportement du producteur.

5 Pour plus de détails sur les conditions de cette enquête sur les caractéristiques de l'échantillon et pour une analyse descriptive approfondie de l'ensemble des variables disponibles, voir Budisantoso (1994).
} 
Tableau 1. Définition des variables

\begin{tabular}{|c|c|c|}
\hline Variable & Définition & $\begin{array}{c}\text { moyenne (\% pour } \\
\text { les variables } \\
\text { muettes) }\end{array}$ \\
\hline Dumvalor & égale à 1 si le ménage est payé suivant la qualité, 0 sinon & $\begin{array}{l}63,48 \\
(0,48)\end{array}$ \\
\hline Prix & prix perçu par lesproducteurs payés suivant la qualité & $\begin{array}{r}1045,07 \\
(93,93)\end{array}$ \\
\hline Prix & prix perçu par les producteurs non payés suivant la qualité & $\begin{array}{r}1027,38 \\
(80,91)\end{array}$ \\
\hline Dotation & dotation en terre par membre de la famille & $\begin{array}{c}0,60 \\
(0,38)\end{array}$ \\
\hline Supcafé & superficie cultivée en café & $\begin{array}{c}1,01 \\
(0,63)\end{array}$ \\
\hline Nomparc & nombre de parcelles caféières & $\begin{array}{c}1,39 \\
(0,63)\end{array}$ \\
\hline Dumcredi ${ }^{\mathrm{i}}$ & égale à 1 si le ménage n'est pas contraint sur le crédit, 0 sinon & $\begin{array}{l}70,44 \\
(0,46)\end{array}$ \\
\hline Dumvente & égale à 1 si le ménage vend le café sur un marché, 0 sinon & $\begin{array}{l}40,87 \\
(0,49)\end{array}$ \\
\hline
\end{tabular}

les chiffres entre parenthèses représentent les écart-types.

Au vu de l'échantillon de producteurs, la première observation à noter est que tous les producteurs ne sont payés à la qualité. Dans l'écriture du modèle théorique précédent, nous avons supposé que la qualité du café était valorisée par une différentiation du prix. Or, dans le système de commercialisation indonésien, il apparaît, dans certains cas, un problème d'information pour des producteurs de café qui ne connaissent pas précisément les exigences des acheteurs du café. Ces derniers peuvent sélectionner sur des critères tels que la couleur des grains de café, le pourcentage de grains abîmés,... Dans ce cas de figure, le producteur ne sait pas avant sa récolte qu'il va être rémunéré suivant la qualité de son produit. Ainsi, dans l'échantillon, 73 producteurs sont payés suivant la qualité de leur café 6 (soit $63 \%$ ) et parmi ce groupe, 56 d'entre eux réalisent une cueillette sélective. Une variable muette notée "dumvalor" permet d'indiquer le groupe où la production est payée suivant la qualité. Dans la spécification du probit, différents produits croisés entre cette variable et certaines caractéristiques de l'exploitation sont introduits.

Dans le modèle théorique, la contrainte de crédit dépend de la variable $\bar{B}$ définissant les garanties du ménage agricole et de la variable $d_{0}$, le montant initial de dettes du ménage. Ces deux variables sont fonctions des ressources en terre du ménage définie par la superficie possédée par membre de la famille par le ménage agricole notée "dotation".

La superficie cultivée en café ("supcafé") influence suivant le modèle théorique la productivité marginale du travail. La prise en compte du nombre de parcelles en café ("nomparc") permet d'introduire l'effet de cette variable sur les coûts fixes associés à la récolte. De plus, une variable muette précise si le ménage est contraint ou non sur le crédit

6 Nous revenons dans le quatrième paragraphe à ce groupe de producteurs non payés à la qualité pour proposer une voie pour réduire les problèmes d'information. 
("dumcred"). Suivant les résultats du modèle théorique, les producteurs payés à la qualité qui font une cueillette non sélective correspondent à des ménages agricoles contraints sur leur montant d'endettement. Pour vérifier ce résultat, nous introduisons dans la spécification du probit le produit croisé entre la variable "dumvalor" et la variable caractérisant l'existence de contrainte de crédit. Enfin, une variable muette précise le lieu de commercialisation du café et notamment permet de définir le groupe de producteurs qui se déplacent pour vendre leur production sur un marché (pour les autres producteurs, ce sont les acheteurs qui viennent au village acheter le café).

Des spécifications alternatives ont été étudiées. Notamment, les caractéristiques individuelles du chef d'exploitation (âge, niveau de formation, expérience caféière) qui peuvent influencer la productivité marginale du travail, ont été introduites pour expliquer la pratique d'exercer une cueillette sélective. Aucune de ces trois variables n'avait un impact significatif.

\subsection{Résultats}

La probabilité d'effectuer une cueillette sélective est estimée sous la forme d'un probit dichotomique par la méthode du maximum de vraisemblance. Les résultats d'estimation sont présentés dans le tableau 1. Ce tableau rapporte également les effets quantitatifs des variables explicatives sur la probabilité d'effectuer une cueillette sélective mesurés par les dérivées partielles. La dérivée partielle associée à une variable mesure la variation de la probabilité de cueillette sélective suite à l'augmentation d'une unité de cette variable. Les dérivées partielle sont calculées au point moyen de l'échantillon.

Tableau 2. Modèle probit d'exercice d'une cueillette sélective

\begin{tabular}{|l|c|c|c|}
\hline Variables explicatives & Coefficients estimés & $\mathrm{t}$ de student & dérivés partielles \\
\hline constante & 0.543 & 0.70 & \\
dotation & -3.252 & -2.19 & -0.465 \\
(dotation) $^{2}$ & 1.542 & 1.97 & \\
supcafé & 0.542 & 1.85 & 0,128 \\
nomparc & -0.518 & -2.17 & $-0,123$ \\
dumvente & 0.685 & 1.95 & 0,171 \\
dumvalor * dumcredi & $-0,698$ & $-1,65$ & $-0,165$ \\
dumvalor * prix & $0.224 \cdot 10^{-2}$ & 3.43 & 0,0006 \\
dumvalor * dotation & -1.602 & -1.61 & $-0,379$ \\
\hline
\end{tabular}

La dotation en terre a un impact négatif sur la probabilité d'effectuer une cueillette sélective. Mais, cet impact n'est pas constant car le terme quadratique de cette variable est significativement différent de zéro. Pour les ménages qui ne vendent pas leur production suivant la qualité, l'impact est négatif pour les agriculteurs possédant moins de 2.11 hectares par habitant, l'effet maximal en valeur absolue intervenant pour des ménages ayant 1.06 hectares par habitant. Autrement dit, une augmentation de la richesse incite les petits agriculteurs à accroître leur endettement. Cet effet provoque un resserrement de la contrainte 
de crédit i.e. augmente la probabilité d'être rationné et par conséquent diminue la probabilité d'effectuer une cueillette sélective. Pour les ménages payés suivant la qualité du café, l'effet négatif de la richesse intervient jusqu'à une dotation égale à 3,15 hectares par personne.

Pour les ménages payés suivant la qualité, une augmentation du prix augmente la probabilité de cueillette sélective i.e. celle de produire du café de bonne qualité. Une spécification alternative a été estimée qui introduisait les variations de prix pour toutes les exploitations mais elles n'avaient pas d'impact significatif. L'effet prix a donc une influence, uniquement, pour le groupe des producteurs payés à la qualité

La superficie cultivée en café augmente la probabilité du phénomène étudié ; cet effet s'explique par l'effet positif de la surface sur la productivité marginale du travail.

Le nombre de parcelles, quant à lui, diminue la probabilité d'exercice d'une cueillette sélective. Le lieu de commercialisation (marché) joue de façon positive sur la probabilité de produire du café de bonne qualité. Cet impact s'explique vraisemblablement par le fait que l'information sur les critères d'achat des acheteurs sont plus perceptibles sur un marché.

En résumé, le tableau 2 montre que l'effet positif d'un accroissement du prix payé à la qualité n'est pas suffisant pour contrecarrer l'effet négatif de la contrainte de crédit. Ces résultats illustrent donc clairement qu'une politique de prix différencié destinée à inciter les producteurs indonésiens à améliorer la qualité du café n'a qu'un impact limité face à des ménages agricoles contraints pour le crédit. Les résultats obtenus corroborent, par conséquent, l'idée couramment admise que la mauvaise qualité observée du café indonésien est essentiellement liée à la situation de dépendance financière des exploitants agricoles vis-à-vis des avances sur récolte consenties traditionnellement par les collecteurs locaux.

Aussi, une voie pour une politique volontariste d'amélioration de la qualité en Indonésie pourrait donc être de rompre ce lien de dépendance financière résultant avant tout d'une longue tradition) entre les paysans et intermédiaires locaux indonésiens en développant un système de crédits institutionnels incitatifs à destination des ménages agricoles. ${ }^{7}$

\subsection{Le rôle de la garantie contractuelle}

Il s'agit ici de proposer une voie alternative pour la politique d'amélioration de la qualité du café en Indonésie. Contrairement à la proposition précédente, cette alternative n'impliquerait pas la rupture du lien de dépendance financière paysans-intermédaires mais une réduction de l'asymétrie d'information existant entre ces deux groupes d'agents.

L'analyse précédente a pu montrer pourquoi la valorisation de la qualité du café n'est pas suffisante pour conduire les producteurs à adopter une cueillette sélective. Ainsi, si les ménages sont contraints par rapport à leur montant d'endettement alors ils ne peuvent pas

7 Le rôle des crédits institutionnels est actuellement quasi-inexistants dans le secteur caféier indonésien (De Graaf, 1986). 
effectuer une cueillette sélective. Pour l'instant, nous avons examiné le cas où le rationnement de crédit est dû à des différences d'information entre les intermédiaires locaux, fournisseurs de crédit et les ménages agricoles, demandeurs de crédit. Pour les prêteurs, il existe ainsi une incertitude sur la qualité de l'emprunteur c'est à dire sur le comportement futur de l'emprunteur en matière de remboursement. Généralement, le plafond de l'endettement réalisable est fonction de la richesse initiale du ménage, ici définie comme la somme des terres possédées par le ménage.

Dans l'échantillon de producteurs utilisé pour l'application économétrique, nous savons qu'il existe des producteurs de café non payés à la qualité. Aussi, indépendamment des éventuelles contraintes de crédit, ces ménages agricoles n'ont aucune incitation particulière à récolter les cerises de café quand elles sont mûres puisqu'ils ne connaissent pas précisément les exigences des acheteurs du café. Pour que ces récolteurs fassent de la cueillette sélective, il faut donc les informer des critères de sélection des cerises de café et définir "une qualité standard". Une solution serait, alors, d'officialiser ces critères qui peuvent être liés aux exigences créées par les importateurs de café indonésien. Cette "institutionnalisation" devant être réalisée par le gouvernement permettait d'annoncer en début de campagne les prix que chaque producteur peut escompter si son café respecte les normes ${ }^{8}$.

De plus, cette définition d'une qualité standard a aussi une influence sur la fonction d'offre de crédit. Ainsi, elle peut permettre de réduire l'asymétrie d'information existant entre les intermédiaires financiers et les emprunteurs en discriminant les producteurs suivant qu'ils soient ou non payés à la qualité. Cette distinction peut se faire en modifiant la définition de la fonction d'offre de crédit. Ainsi, dans ce cas, l'offre de crédit dépend non seulement de la terre possédée par le ménage mais aussi du rapport des prix. Cette nouvelle définition de la garantie peut devenir une incitation supplémentaire à produire du café de bonne qualité. L'impact du changement dans la définition de cette caution peut être facilement illustrée en adoptant des spécifications particulières pour la fonction d'offre de crédit.

On examine deux cas: i) le cas où il n'existe pas d'annonce avant la campagne sur le système de prix et ii) le cas alternatif où il y a une reconnaissance officielle de la qualité du café.

Dans le premier cas, l'offre de crédit dépend des ressources du ménage, en l'occurrence on suppose qu'elle dépend linéairement du niveau des terres possédées. Ainsi, l'offre s'écrit $B(A)=a A$.

Dans le second cas, l'offre de crédit dépend aussi du système de prix. Pour tenir compte du rapport des prix on retient une forme multiplicative. Ainsi, l'offre se définit par $B\left(A, p_{2} / p_{1}\right)=a A\left(p_{2} / p_{1}\right)$.

Une simulation est effectuée pour mesurer l'impact de la prise en compte des différences de prix dans la définition du plafond de crédit possible. Pour le paramètre $a$ on

8 Actuellement, quand les producteurs sont payés à la qualité le prix perçu est fixé par son intermédiaire. 
retient la valeur 2, valeur utilisée dans différentes études appliquées à des agricultures de pays en voie de développement (Feder, 1985). Pour les valeurs des prix, on utilise les caractéristiques de l'échantillon. Ainsi, pour le prix $p_{2}$ on retient le prix maximal reçu par les producteurs de café payés à la qualité et effectuant une cueillette sélective. Il est égal à 1275 rupiahs par kilos. Pour le prix $p_{I}$ on retient la valeur perçue par les producteurs non payés à la qualité et n'effectuant pas de cueillette sélective qui est égale à 925 rupiahs par kilos. On prend le montant initial de l'endettement égal à 1 pour toutes les exploitations. Dans les deux cas, on détermine pour chaque ménage le montant du niveau maximal de l'endettement autorisé. Un résumé statistique de ces garanties est fourni dans le tableau 3.

Tableau 3. Modification de la contrainte de crédit

\begin{tabular}{|l|c|c|c|}
\hline & moyenne & valeur minimale & valeur maximale \\
\hline$B_{I}$ & 1,20 & 0,25 & 4,63 \\
$B_{2}$ & $\begin{array}{c}(0,76) \\
1,65\end{array}$ & 0,35 & 6,38 \\
\hline
\end{tabular}

Alors dans le premier cas de figure $53 \%$ des producteurs sont contraints contre $35 \%$ dans le second cas. Le fait de réduire cette asymétrie d'information permet de diminuer le nombre de producteurs contraints et de favoriser la probabilité de faire une cueillette sélective.

\section{Conclusion}

L'objectif de cet article consiste, tout d'abord, en la définition d'un cadre théorique permettant de comprendre le comportement de cueillette sélective conditionnant la qualité du café indonésien. De plus, l'objectif appliqué est de proposer des solutions qui permettraient d'inciter les producteurs de café à réaliser la cueillette sélective c'est à dire à ne récolter que les cerises de café mûres.

Le modèle théorique permet de démontrer que l'origine principale des problèmes de qualité de la production de café en Indonésie réside dans le rationnement du crédit subi par les ménage agricoles. Dans ce cas, une politique de prix différencié valorisant le café de bonne qualité n'est pas suffisante pour inciter les producteurs à décaler la date de récolte et donc à réaliser la cueillette sélective. L'estimation économétrique a permis de montrer qu'une augmentation de la richesse incite les petits agriculteurs à accroître leur endettement c'est à dire à resserrer leur contrainte de crédit et donc à diminuer la probabilité de produire du café de bonne qualité .

L'origine de ce rationnement est dû à l'asymétrie d'information qui peut apparaître à la fois du côté de l'intermédiaire financier et du côté du producteur. La politique de différentiation de prix engagée par le gouvernement indonésien doit donc être complétée pour favoriser le développement d'une production de qualité.

Deux axes possibles au niveau politique d'intervention ont pu être dégagés pour favoriser une production de bonne qualité. Aussi, une solution pour une politique volontariste 
d'amélioration de la qualité en Indonésie pourrait être de rompre le lien de dépendance financière entre les paysans et intermédiaires locaux indonésiens en développant un système de crédits institutionnels incitatifs à destination des ménages agricole. La deuxième solution est d'officialiser les critères de qualité de café pour diminuer le rationnement du crédit des ménages. 


\section{Références}

Budisantoso N., (1994), La politique et la stratégie commerciale du café indonésien dans son environnement international, thèse Ecole nationale Supérieure Agronomique de Rennes, 394 pages.

Budisantoso N., Le Mouël C. (1994), Les dilemmes de la politique indonésienne d'exportation du café dans "Economie des politiques agricoles dans les pays en développement" Editions de la Revue Française d'Economie, pp. 43-83.

De Graaf J. (1986), Coffee in Indonesia dans "The economics of coffee economics of crop in developing counties", $\mathrm{n}^{\circ} 1$, Pudoc Wageningen.

Feder G., Lau Lau, L. Lin J. et Luo X. (1990) The relationship between Credit and productivity in Chinese agriculture: a microeconomic model of disequibrium, American Journal of Agricultural Economics, .vol.72, n 5, 1151-57.

Feder, G., (1985) The relationship between farm size and farm productivity : the role of family labor, Supervision and Credit constraints, Journal of development economics, $\mathrm{n}^{\circ} 18,297-313$.

Hardjosuwito, Hermansyah (1984), Le grain de café vert évalué par le système défaut, revue menari Perkebunan, $\mathrm{n}^{\circ} 53$.

Judge G.G., Griffiths W.E., Hill R.C., Lutkepohl H. and Lee T.C., (1985), The Theory and Practice of Econometrics, 2nde edition, New-York, Wiley.

Nakajima C., (1966), Subjective Equilibrium Theory of the Farm, Elsevier, Amsterdam.

Phimister E., (1993) Savings and investment in Farm Households, Avebury, 187 p.

Rajino A.Y., (1985), Amélioration de la qualité de café en raison d'augmenter le revenu des paysans, papier présenté dans "Temu Karya Kopi III", Surabaya 21-23 mai, BPP Bogor, Majalah Kopi Indonesia $n^{\circ} 12$, AEKI-Jakarta.

Singh (I. J.), Squire (L.) et Strauss (J.), (1986) - Agricultural household models : extensions, applications and policy, Published for the World bank, The John Hopkins University Press, Baltimore and London, 335 p.

Stigliss et Weiss, (1983) Credit Rationing in markets with imperfect information, American Economic Review, vol.71, n³, 393-410. 